\title{
FIBRA DIETARIA: UNA ALTERNATIVA \\ PARA LA ALIMENTACIÓN
}

\author{
RAFAel Villanueva Flores* \\ Universidad de Lima
}

Recibido: 9 de febrero del 2018 / Aprobado: 4 de diciembre del 2018

doi: 10.26439/ing.ind2019.n037.4550

RESUMEN: La fibra dietaria está compuesta por un conjunto de elementos con características diferentes que le proporcionan distintas propiedades físicoquímicas, que determinan efectos fisiológicos definidos. Su consumo trae beneficios para la salud y está por debajo de lo recomendado en el Perú y en muchos países del mundo, lo cual es una oportunidad para la industria de alimentos del Perú de desarrollar productos de consumo masivo con elevado contenido de fibra.

Palabras clave: fibra / nutrición / beneficios para la salud /industria de alimentos

\section{DIETARY FIBER:}

\section{AN ALTERNATIVE FOR NUTRITION}

ABSTRACT: Dietary fiber consists of a set of elements with different characteristics that provide several physical and chemical properties that determine clear physiological effects. Its consumption provides health benefits; however, it is below the recommended level both in Peru and many countries worldwide. This situation brings an opportunity for the Peruvian food industry to develop mass consumption products with high fiber content.

Keywords: fiber / nutrition / health benefits / food industry

*RVillan@correo.ulima.edu.pe. 


\section{INTRODUCCIÓN}

El término fibra dietaria se utilizó por primera vez en 1953 para describir los componentes de las paredes celulares de las plantas no digeribles por el ser humano (DeVries, Prosky, Li y Cho, 1999; Jones, 2013).

La fibra dietaria incluye a un rango diverso de carbohidratos compuestos que juegan un rol protagónico en el análisis de la salud pública, por ser un nutriente consumido en menor cantidad a lo recomendado en la mayoría de países del mundo (Millen et al., 2016). No hay duda de que una dieta rica en granos enteros $y$, por ende, en fibra, protege de la obesidad (Kyrø et al., 2011), reduce el riesgo de contraer ciertas enfermedades, como diabetes tipo 2 (Pan et al., 2010), enfermedades cardiovasculares (Mellen, Walsh y Herrington, 2008) y ciertos cánceres del tracto digestivo (Fardet, 2013). Por otro lado, dietas bajas en fibra aumentan el riesgo de enfermedades e impactan negativamente en la funcionalidad del intestino (Brouns, Delzenne y Gibson, 2017).

Sin embargo, algunas fibras específicas y moléculas de carbohidratos no absorbibles pueden conducir a sensaciones intestinales desagradables como resultado de cambios de fluido osmótico o relativa fermentación rápida por la microflora intestinal. Estos carbohidratos son oligosacáridos, disacáridos y polioles rápidamente fermentables (Shepherd, Lomer y Gibson, 2013). Las molestias intestinales parecen exacerbarse en personas que sufren de hipersensibilidad intestinal, comúnmente conocida como síndrome de intestino irritable (Halmos, Power, Shepherd Gibson y Muir, 2014).

\section{MARCO TEÓRICO}

\subsection{Definición de fibra dietaria}

La fibra dietaria es la parte comestible de las plantas o hidratos de carbono análogos que son resistentes a la digestión y absorción en el intestino delgado, con fermentación completa o parcial en el intestino grueso (ACC International, 2001).

Según la comisión de Codex Alimentarius (2010), la fibra dietaria consiste en polímeros de carbohidratos con diez o más unidades monoméricas, que no son hidrolizados por enzimas endógenas en el intestino delgado de los seres humanos y que pertenecen a las siguientes categorías:

- Polímeros de carbohidratos comestibles que se producen naturalmente en los alimentos.

- Polímeros de carbohidratos que se han obtenido de materia prima alimenticia por medios físicos, enzimáticos o químicos que poseen algún efecto fisiológico beneficioso para la salud. 
- Polímeros de carbohidratos sintéticos con algún efecto fisiológico beneficioso para la salud.

Sin embargo, la gran controversia sobre la definición se refiere a la inclusión o no de los oligosacáridos con grados de polimerización (DP) en el rango de 3 a 9 (como inulina, fructooligosacáridos, galactoolisacáridos, maltodextrinas resistentes, rafinosa y otros), decisión que, para efectos de rotulado nutricional, pueden tomar individualmente las autoridades de salud competentes de cada país (Howlett et al., 2010).

\subsection{Clasificación de la fibra dietaria}

La Administración de Alimentos y Medicamentos (FDA por sus siglas en inglés) clasificó en el 2016 a la fibra dietaria en dos categorías:

- Fibras intrínsecas e intactas: esencialmente componentes intactos de plantas. Se encuentran en alimentos tales como vegetales, granos integrales, frutas, salvado de cereales, cereal en hojuelas y harinas. Las fibras también se consideran intactas porque no se han eliminado de los alimentos. Se ha demostrado que los alimentos que contienen estas fibras son beneficiosos y los fabricantes no necesitan demostrar que proporcionan efectos fisiológicos beneficiosos para la salud humana.

- Fibras sintéticas y aisladas: utilizadas principalmente para fortificar alimentos. Los fabricantes que usan fibras que se agregan a los alimentos, ya sea aislándolas de otros alimentos o sintetizándolas, deben demostrar que poseen efectos fisiológicos beneficiosos para la salud humana para cumplir con la definición de fibra dietaria. Entre las más utilizadas tenemos a la goma acacia, goma guar, goma de algarrobo, celulosa, carboximetilcelulosa, pectina, beta glucano soluble, inulina, fibra de manzana, fibra de bambú, fibra de semilla de algodón, fibra de leguminosas, goma karaya, polidextrosa, fibra de papa, fibra de soya, almidón de maíz con elevado contenido de amilosa, almidones resistentes de trigo y maíz, fibra de caña de azúcar, fibra de remolacha, goma xantan.

\section{MÉTODOS DE DETERMINACIÓN DE FIBRA DIETARIA}

El Codex Alimentarius (1997) define cuatro clases de métodos para la determinación de fibra dietaria, cada uno de ellos con su propio rango de aplicación:

- Tipo I (método de definición): determina un valor al cual solo se puede llegar mediante el método per se y se utiliza por definición como única metodología para determinar el valor aceptable del ítem medido. 
- Tipo II (método de referencia): definido como método referencial cuando el tipo I no puede ser aplicado. Debe ser recomendado para utilizarse en casos de disputas o calibraciones.

- Tipo III (método alternativo aprobado): cumple con los requisitos del comité del Codex para análisis y muestreo y puede ser utilizado para control, inspecciones $\mathrm{y}$ aspectos regulatorios.

- Tipo IV (método tentativo): es el que ha sido utilizado tradicionalmente o que ha sido introducido recientemente pero no ha sido aceptado por la comisión del Codex.

\section{ANÁLISIS}

\subsection{Consumo recomendado}

Escudero y González (2006) recomiendan una ingesta rica en fibra desde los primeros años de la vida ya que a menudo va acompañada de un estilo de vida que, a largo plazo, ayuda a controlar otros factores de riesgo. El consumo recomendado de la fibra dietaria depende de la edad, sexo y cantidad de energía ingerida. En general el consumo diario de la fibra dietaria debe estar en el rango de 18 a $38 \mathrm{~g} /$ día para personas adultas (Vilcanqui y Vílchez, 2017).

Las Guías Alimentarias de los años 2005 y 2010 del Departamento de Agricultura de los Estados Unidos (USDA por sus siglas en inglés) identificaron a la fibra dietaria como un nutriente a tener en cuenta debido a su bajo consumo (USDA, 2005, 2010) por lo cual la dosis diaria recomendada fue aumentada de 25 a $28 \mathrm{~g}$ para una dieta diaria de $2000 \mathrm{kcal}$ (USDA, 2016). Más aún, la guía del año 2015 cambió de un modelo reduccionista de interpretación de la ciencia de la nutrición (analizando nutrientes aislados bajo situaciones controladas) a uno basado en entender patrones dietéticos (USDA, 2015).

El Panel de Productos Dietéticos, Nutrición y Alergias de la Autoridad Europea de Seguridad Alimentaria (EFSA por sus siglas en inglés) estableció en el 2010 que para el normal funcionamiento del intestino grueso es necesario un consumo diario de 25 g de fibra dietética en adultos (Agencia Española de Consumo, Seguridad Alimentaria y Nutrición, 2010). Canadá establece un valor de referencia diario de $25 \mathrm{~g}$ de fibra para mujeres adultas y de $38 \mathrm{~g}$ para hombres, resaltando que los canadienses consumen la mitad de fibra de lo recomendado (Gobierno de Canadá, 2017). Más aún, se ha calculado que si las personas adultas en Canadá aumentasen en un gramo por día el consumo de fibra dietaria, los gastos anuales en salud relacionados a enfermedades cardiovasculares y diabetes tipo 2 podrían reducirse en CAD 143,2 millones (Abdullah, Gyles, Marinangeli, Carlberg y Jones, 2015). 
La Asociación Americana de Diabetes (ADA por sus siglas en inglés) recomienda un consumo de fibra entre 20 y $35 \mathrm{~g}$ por día, tanto soluble como insoluble, para mantener un mejor control glucémico e insulínico (Asociación Mexicana de Diabetes, s. f.).

En el Perú, el consumo de fibra es 50 \% de lo recomendado (Lázaro, 2013), lo cual abre una buena alternativa para la industria de alimentos de desarrollar productos con elevado contenido de fibra. Más aún cuando más del 35,5 \% de la población peruana menor a 15 años tiene sobrepeso y $17,8 \%$ obesidad (INEI, 2015).

\subsection{Regulación para rotulado de alimentos}

La fibra dietaria que puede declararse en la etiqueta dentro de la información nutricional incluye ciertas fibras naturales que son intrínsecas e intactas en las plantas, carbohidratos solubles e insolubles no digeribles aislados añadidos, que la autoridad sanitaria de cada país debe determinar que poseen efectos fisiológicos beneficiosos para la salud humana o que resulta de una ingesta reducida de calorías y mejora en la función intestinal.

La definición de fibra dietaria del Codex promueve el concepto de que compuestos que se comportan en vivo como fibra, independientemente de la fuente, pueden ser incluidos como fibra dietaria en el rotulado nutricional, siempre que se demuestren los beneficios fisiológicos y a la salud (Zielinski, DeVries, Craig y Bridges, 2013). Muchos países, basados en evidencias científicas, incluyen a los oligosacáridos con DP $\geq 3$ dentro de su definición de fibra dietaria (De Menezes et al., 2013).

La EFSA, la Autoridad Estatutaria de Estándares Alimentarios de Australia y Nueva Zelanda, Health Canada y las agencias equivalentes en China, Japón, Brasil, Chile, México, Tailandia, Corea, Singapur, Malasia e Indonesia, han adoptado la definición dada por el Codex (que incluye a los carbohidratos con DP 3-9). Sudáfrica es el único país que ha decidido no incluir a los oligosacáridos con DP 3-9 en su definición de fibra dietaria (Dai y Chau, 2017). En el Perú no hay regulación al respecto y el etiquetado nutricional no es obligatorio, con excepción de regímenes especiales como productos infantiles. La normativa aplicable al Perú en materia de etiquetado puede resumirse en las siguientes regulaciones:

- Decreto Supremo 007-98-SA: reglamento sobre vigilancia y control sanitario de alimentos y bebidas y sus modificatorias.

- Norma Metrológica Peruana 001-2014: etiquetado de productos preenvasados.

- Ley 28405: ley de rotulado de productos industriales manufacturados (sin reglamento).

- Ley 29571: código de protección y defensa del consumidor. 
- Decreto Supremo 009-2006-SA: reglamento de alimentación infantil.

- Ley 28814: reglamento que dispone la fortificación de la harina de trigo con micronutrientes.

- Decreto Supremo 004-2006-AG: reglamento técnico para los productos orgánicos o ecológicos. Ley 29196 de promoción de la producción orgánica o ecológica.

- Ley 30021: ley de promoción de la alimentación saludable para niños, niñas y adolescentes.

- $\quad$ Decreto Supremo 007-2017-MINAGRI que aprueba el reglamento de la leche y productos lácteos.

\subsection{Fuente de fibra dietaria}

Tradicionalmente los consumidores han identificado a los granos enteros, nueces, frutas y vegetales como fuente principal de fibra dietaria. El trigo, el arroz y el maíz son los granos enteros más consumidos en el mundo, seguidos por la avena, el centeno y la cebada. Los granos enteros contienen elevada cantidad de sustancias bioquímicas, como fibra, vitaminas, minerales, antioxidantes, fitoquímicos como betaína, cloro, aminoácidos sulfurosos y melatonina, que representan al menos el $15 \%$ del peso del grano (Fardet, 2013).

Sin embargo, la industria de alimentos ha introducido al mercado productos con diferentes niveles de fibra dietaria en su composición. Los ingredientes utilizados poseen propiedades únicas que elevan el contenido de fibra y adicionalmente poseen otras funciones en la formulación del producto (Nelson, 2002).

Cuando mencionamos a la fibra dietaria nos referimos tanto a fibra soluble como a insoluble. La fibra soluble se disuelve en agua para formar en el estómago una sustancia gruesa de gran viscosidad similar a un gel. Se descompone por bacterias en el intestino grueso y proporciona algunas calorías. La fibra insoluble no se disuelve en agua y pasa a través del tracto gastrointestinal relativamente intacta en forma de una mezcla de baja viscosidad y, por lo tanto, no es fuente de calorías (FDA, 2017).

Como fuente de fibra insoluble tenemos a la celulosa (trigo integral, salvado de trigo, hortalizas), hemicelulosa (cereales, hortalizas, salvado), lignina (hortalizas, frutas), cutina, suberina, ceras de plantas, quitina, quitosano y almidones resistentes, y como fuente de fibra soluble a pectinas (frutas, hortalizas), $\beta$-glucanos (cebada, avena), gomas (avena, cebada, chía) e inulinas.

El término almidón resistente fue adoptado a principios de la década de 1980 por Hans Englyst, un fisiólogo británico, y se define como almidón dietético que no se digiere 
en el intestino delgado (Saura-Calixto, Goñi, Bravo y Manas, 1993). El almidón resistente, por lo tanto, se comporta como fibra dietaria y puede tener potencial como un ingrediente favorable para la salud. La principal diferencia entre los almidones digeribles y resistentes es la accesibilidad del almidón a los procesos digestivos y, posteriormente, la facilidad con la que se pueden cortar los enlaces glicosídicos contenidos en las moléculas de almidón (Stephen, 1995). El almidón resistente puede ser encontrado en granos y semillas parcialmente molidos, en plátanos verdes y en la papa, tanto cruda como cocida.

Las leguminosas (frijol, arveja, habas, lentejas, pallar, lupino) y sus derivados son una excelente fuente de fibra dietaria. Media taza de menestras proporciona, aproximadamente, la tercera parte del requisito diario de fibra.

\subsection{Beneficios para la salud}

Hipócrates dijo alguna vez que "el pan integral generaba heces más grandes que el pan refinado", resaltando la importancia de la fibra en términos de sus beneficios fisiológicos para la salud como el alivio del estreñimiento (Dai y Chau, 2017).

La fibra dietaria se convirtió, entre los años 1960 y 1970, en un concepto nutricional importante debido a las observaciones de los médicos británicos Burkitt, Painter, Walker y Trowell, que indicaban que ciertas enfermedades, como diabetes, cardiopatías y cáncer de colon, que se veían regularmente en los países occidentales eran raramente vistas en el este del África rural. Lo atribuyeron específicamente a la marcada diferencia en la ingesta de carbohidratos no refinados. Con ello surgió la hipótesis de la fibra dietaria que señalaba que desde el estreñimiento hasta la enfermedad coronaria podían reducirse con la adición de fibra dietética a la dieta (DeVries, Prosky, Li y Cho, 1999; Jones, 2013).

La fibra participa en todas las funciones del sistema digestivo, desde la masticación hasta la evacuación de las heces (Escudero y González, 2006). En general los beneficios para la salud que proporciona la fibra dietaria incluyen mejoras en la salud intestinal (laxación, disminución de tiempo de tránsito, aumento de carga fecal, ablandado de heces, disminución de $\mathrm{pH}$ fecal y fermentación), control glicémico (glucosa en la sangre y atenuación de la insulina), reducción de colesterol (colesterol total y colesterol LDL), control de peso (reducción de ingesta calórica y aumento de saciedad) y aumento de absorción de minerales (Jones, 2013; Grabitske y Slavin, 2009). Se estima que el rol en la reducción del colesterol está relacionado con la capacidad para unir los ácidos biliares en el intestino delgado, causando que sean excretados en lugar de ser reabsorbidos en el hígado. Dado que el colesterol se convierte en ácidos biliares en el hígado, la unión y la excreción de ácidos biliares causa un aumento en la conversión de colesterol a ácidos biliares, disminuyendo así el grupo de colesterol (Nelson, 2002). 
Recordemos que las fibras insolubles (presentes en la parte externa de semillas y granos, salvado de trigo, maíz, cereales integrales, en las cáscaras de las manzanas y peras, en la parte blanca de las frutas cítricas y legumbres) no son digeridas ni en el estómago ni en el intestino delgado pero pueden ser fermentadas a cierto nivel por bacterias del colon en el intestino grueso, mientras que las fibras solubles (que se encuentran principalmente en las frutas y verduras, especialmente en manzanas, naranjas, zanahorias, brócoli y cebollas, al igual que en el salvado de avena, cebada, nueces, almendras, avellanas y legumbres) escapan a la digestión del estómago y del intestino delgado pero son fermentadas rápidamente en el intestino grueso.

La acelerada investigación en microbioma intestinal ha abierto una nueva perspectiva del rol de la fibra dietaria en la salud humana. El microbioma impacta prácticamente en cualquier sistema del cuerpo y la fibra es la primera fuente de nutrición. Esta área de investigación es posible por la tecnología secuencial del genoma, que nos ayuda a decodificar la composición y función de las bacterias intestinales (Birket y Brouns, 2017).

La Organización Mundial de la Salud (OMS) recomienda el consumo de cinco piezas o porciones $(400 \mathrm{~g})$ de frutas y verduras al día para reducir el riesgo de desarrollar enfermedades no transmisibles y ayudar a garantizar una ingesta diaria suficiente de fibra dietética (OMS, 2018).

\subsection{Productos con elevado contenido de fibra}

La fibra puede, durante el procesamiento de alimentos, agregar volumen, aumentar viscosidad, formar geles (por su capacidad de retención y enlace de agua) y reemplazar o imitar a las grasas (Nelson, 2002).

El tipo de procesamiento utilizado para producir ingredientes con alto contenido de fibra influye en gran medida en la funcionalidad del ingrediente final. Procesos como molienda, blanqueado, refinado, tratamientos enzimáticos, procedimientos de estabilización, extrusión, deshidratación o secado y tostado son algunos de los que se utilizan en la producción de ingredientes ricos en fibra.

De las diferentes categorías de alimentos, los más comunes enriquecidos con fibra son los productos horneados y extruidos a partir de granos que, con el desarrollo de la tecnología y los ingredientes, han logrado una textura, sabor y apariencia aceptados por los consumidores. Dentro de estos productos tenemos a los panes, panetones, galletas, fideos, hojuelas, pizzas y en menor proporción, bizcochos, obleas, muffins, dado que estos últimos contienen más agua en su formulación en comparación con panes y galletas. Debemos tener en cuenta que en panificación la fibra aumenta la absorción de agua durante la etapa de mezclado, incrementa el tiempo de mezclado modificando la reología de la masa por lo cual las condiciones del proceso deben ser adaptadas. También modifica el color y textura del producto y reduce el volumen. 
Bebidas de frutas, tés y batidos son productos en los cuales se pueden adicionar vitaminas, minerales, antioxidantes y fibra para mejorar su capacidad nutritiva. En estos productos, la fuente principal de fibra soluble proviene de pectina, goma arábiga e inulina, debido a su facilidad de dispersión en agua.

También pueden desarrollarse productos lácteos con fibra, como yogures, pudines, helados, quesos y productos relacionados. Para estas aplicaciones se recomienda el uso de fibras solubles en agua por su capacidad para ligar agua y su baja detección en el producto final.

El agente gelificante más utilizado en la producción de mermeladas es la pectina, la cual es un ingrediente rico en fibra. Sin embargo, su uso en niveles bajos (típicamente menos del $1 \%$ ) en mermeladas no contribuye significativamente en el contenido de fibra del producto.

Los ingredientes con elevado contenido de fibra utilizados en sopas y salsas sirven para espesar o para suspender especies. Los almidones son los agentes espesantes más comunes, usados en sopas y salsas, mientras que las gomas hidrocoloides son las más utilizadas en aderezos. Se pueden utilizar fibras solubles en agua para reemplazar la grasa, mejorar la sensación bucal y aumentar la viscosidad, mientras que fibras insolubles como celulosa, por su capacidad de retener agua y aceite, se pueden utilizar para mejorar su estabilidad.

\section{CONCLUSIONES}

El consumo de fibra dietaria trae beneficios para la salud y su consumo en el Perú y en muchos países del mundo es menor a lo recomendado. El bajo consumo de fibra se puede explicar por una mayor oferta y consumo de productos refinados. La industria de alimentos en el Perú tiene una gran oportunidad de desarrollar e introducir en el mercado productos de consumo masivo con elevado contenido de fibra. Esto puede ser reforzado por campañas de entidades reguladoras de salud, resaltando los beneficios del consumo de fibra en la salud de la población, lo que también podría llevarnos obtener ahorros en el tratamiento de enfermedades cardiovasculares y diabetes tipo 2.

\section{REFERENCIAS}

Abdullah, M. M., Gyles, C. L., Marinangeli, C. P., Carlberg, J. G. y Jones, P. J. (2015). Costof-illness analysis reveals potential healthcare savings with reductions in type 2 diabetes and cardiovascular disease following recommended intakes of dietary fiber in Canada. Front Pharmaco 6, pp. 1-12. doi: 10.3389/fphar.2015.00167 
Agencia Española de Consumo, Seguridad Alimentaria y Nutrición (25 de marzo del 2010). EFSA establece valores dietéticos de referencia para el consumo de nutrientes. Recuperado de http://www.aecosan.msssi.gob.es/AECOSAN/web/seguridad_ alimentaria/noticias_efsa/2010/valores_nutrientes.htm.

American Association of Cereal Chemists International (2001). Definition of dietary fiber. Cereal Foods World 46(3), pp. 112-125.

Asociación Mexicana de Diabetes (s. f.). ¿Es la fibra dietética importante para el control de la glucosa? Recuperado de: http://amdiabetes.org/archivos/alimentos-articulos/ la-fibra-dietetica-importante-control-la-glucosa

Birkett, A. y Brouns, F. (2017). Why Is Fiber a Hot Nutrient? Cereal Food Word 63(2), pp. 62-63. doi:10.1094/CFW-62-3-0087

Brouns, F., Delzenne, N. y Gibson G. (2017). The Dietary Fibers-FODMAP's Controversy. Cereal Foods World 62(3), pp. 98-103. doi: 10.1094/CFW-62-3-0098

Codex Alimentarius (1997). Principles for the establishment of Codex methods of analysis. Recuperado de http://ww.fao.org/docrep/w5975e/w5975e09.htm.

Codex Alimentarius (2010). Guidelines on nutrition labelling CAC/GL 2-1985. Roma: FAO.

Dai, F. G. y Chau, C. F. (2017). Classification and regulatory perspectives of dietary fiber. Journal of Food and Drug Analysis 25(1), pp. 37-42. doi: 10.1016/j.jfda.2016.09.006

De Menezes, E. W., Giuntini, E. B.,Dan, M. C., Sardá, F. A. y Lajolo, F. M. (2013). Codex dietary fibre definition - Justification for inclusion of carbohydrates from 3 to 9 degrees of polymerization. Food Chemistry 140(3), pp. 581-585. doi: 10.1016/j. foodchem.2013.02.075

Decreto Supremo 007-98-SA (24 de septiembre de 1998). Decreto legislativo que aprueba el reglamento sobre vigilancia y control sanitario de alimentos y bebidas y sus modificatorias. Recuperado de https://www.gob.pe/institucion/minsa/ normas-legales/256394-007-98-sa

Decreto Supremo 009-2006-SA (16 de junio del 2006). Decreto legislativo que aprueba el reglamento de alimentación infantil. Recuperado de http:// www.sipi.siteal.iipe.unesco.org/normativas/188/decreto-supremo-ndeg0092006-reglamento-de-alimentacion-infantil

Decreto Supremo 004-2006-AG (14 de julio del 2006). Decreto que aprueba el reglamento técnico para los productos orgánicos o ecológicos. Recuperado de https://www. senasa.gob.pe/senasa/normas-sobre-produccion-organica/

Decreto Supremo 007-2017 (28 de septiembre del 2017). Decreto legislativo que aprueba el reglamento de la leche y productos lácteos. Recuperado de 
https://www.minagri.gob.pe/portal/decreto-supremo/ds-2017/19598 -decreto-supremo-n-007-2017-minagri

DeVries, J. W., Prosky, L., Li, B. y Cho, S. (1999). A historical perspective on defining dietary fiber. Cereal Foods World 44(6), pp. 367-369.

Escudero Álvarez, E. y González Sánchez, P. (2006). La fibra dietética. Nutrición Hospitalaria 21(2), pp. 61-72.

Fardet, A. (2013). Whole Grains from a Mechanistic View. Recuperado de https://www. aaccnet.org/publications/plexus/cfwplexus/library/books/Documents/ WholeGrainsSummit2012/CPLEX-2013-1001-01B.pdf

Fundación Española del Corazón (7 de abril de 2011). La fibra nos hace vivir más... iy mejor! Recuperado de http://www.fundaciondelcorazon.com/corazon-facil/ blog-impulso-vital/2081-fibra-vivir-mas-mejor.html.

Gobierno de Canadá (2017). Fibre. Recuperado de https://www.canada.ca/en/healthcanada/services/nutrients/fibre.html.

Grabitske, H. M. y Slavin J. L. (2009). Gastrointestinal effects of low-digestible carbohydrates. Critical Reviews in Food Science and Nutrition 49(4), pp. 327-60.

Halmos, E. P., Power, V. A., Shepherd, S. J., Gibson, P. R. y Muir, J. G. (2014). A diet low in FODMAPs reduces symptoms of irritable bowel syndrome. Gastroenterology 146(1), pp. 67-75.

Health Canada (2012). Do Canadian Adults Meet Their Nutrient Requirements Through Food Intake Alone? Recuperado de https://www.canada.ca/en/health-canada/ services/food-nutrition/food-nutrition-surveillance/health-nutrition-surveys/ canadian-community-health-survey-cchs/canadian-adults-meet-theirnutrient-requirements-through-food-intake-alone-health-canada-2012.html.

Howlett, J. F., Betteridge, V. A., Champ, M., Craig, S. A. S., Meheust, A. y Miller-Jones, J. (2010). The definition of dietary fiber: Discussions at the ninth Vahouny fiber symposium: Building scientific agreement. Food \& Nutrition Research 54(5750). doi: $10.3402 /$ fnr.v54i0.5750

Instituto Nacional de Estadística e Informática (INEI) (2015). Encuesta demográfica y de salud familiar. Recuperado de http://www.inei.gob.pe/media/MenuRecursivo/ publicaciones_digitales/Est/Lib1356/

Instituto de Nacional de Salud (INS). (2009). Tablas peruanas de composición de alimentos. Lima: Ministerio de Salud. Recuperado de http://www.ins.gob.pe/insvirtual/ images/otrpubs/pdf/Tabla\%20de\%20Alimentos.pdf 
Jones, J. M. (2013). Dietary Fiber Future Directions: Integrating New Definitions and Findings to Inform Nutrition Research and Communication. Advances in Nutrition 4(1), pp. 8-15.

Kyrø, C., Skeie G., Dragsted, L. O., Christensen, J., Overvad, K., Hallmans, G., ... Olsen, A. (2011). Intake of whole grains in Scandinavia is associated with healthy lifestyle, socio-economic and dietary factors. Public Health Nutrition First Review 10, pp. 1-9. doi: $10.1017 /$ S1368980011000206

Lázaro, M. (2013). Guías alimentarias para la población peruana. Avances 2013. Lima: Ministerio de Salud.

Ley 28405 (19 de noviembre del 2004) Ley de rotulado de productos industriales manufacturados (sin reglamento). Lima.

Ley 28814 ( 25 de julio de 2006). Ley que dispone la fortificación de la harina de trigo con micronutrientes. Lima. Recuperado de https://www.saludarequipa.gob.pe/

Ley 29571 (2 de septiembre del 2010). Código de protección y defensa del consumidor. Lima. Recuperado de http://www.leyes.congreso.gob.pe/

Ley 29196 (24 de julio del 2012). Ley de promoción de la producción orgánica o ecológica. Recuperado de https://www.senasa.gob.pe/senasa/

Ley 30021 (17 de mayo del 2013). Ley de promoción de la alimentación saludable para niños, niñas y adolescentes. Lima. Recuperado de http://www.leyes.congreso.gob.pe/

Mellen, P. B., Walsh, T. F. y Herrington, D. M. (2008). Whole grain intake and cardiovascular disease: a meta-analysis. Nutrition, Metabolism and Cardiovascular Diseases 18(14), pp. 283-290.

Millen, B. E., Abrams, S., Adams-Campbell, L., Anderson, C., Brenna, J. T., Campbell, W., ... Lichtenstein, A. H. (2016). The 2015 Dietary Guidelines Advisory Committee Scientific Report: Development and Major Conclusions. Advances in Nutrition 7, pp. 438-444.

Murphy, N., Norat, T., Ferrari, P., Jenab, M., Bueno-de-Mesquita, B., Skeie, G., ... Riboli, E. (2012). Dietary fibre intake and risks of cancers of the colon and rectum in the European prospective investigation into cancer and nutrition (EPIC). PLoS One 7(6), pp. 1-10. doi: 10.1371/journal.pone.0039361

Nelson, A. L. (2002). High Fiber Ingredients. Minnesota: American Association of Cereal Chemists Inc.

Norma Metrológica Peruana 001-2014 (29 de diciembre dl 2014) Etiquetado de productos pre envasados. (29 de diciembre del 2014). Lima. Recuperado de https://www. indecopi.gob.pe/documents 
Organización Mundial de la Salud (OMS) (31 de agosto de 2018). Alimentación sana. Nota descriptiva N. ${ }^{\circ}$ 394. Recuperado de: http://www.who.int/mediacentre/ factsheets/fs394/es/.

Pan, A., Lucas, M., Sun, Q., Van Dam, R. M., Franco, O. H., Manson, J. E., ... Hu, F. B. (2010). Bidirectional association between depression and type 2 diabetes mellitus in women. Archives of International Medicine. JAMA Internal Medecine 170(21): pp. 1884-1891. doi: 10.1001/archinternmed.2010.356

Saura-Calixto, F., Goñi, I., Bravo, L. y Manas, E. (1993). Resistant starch in foods: Modified method for dietary fiber residues. Journal of Food Science 58(3), pp. 642-643.

Shepherd, S. J., Lomer, M. C. y Gibson, P. R. (2013). Short-chain carbohydrates and functional gastrointestinal disorders. American Journal of Gastroenterology 108(5), pp. 707-717. doi: 10.1038/ajg.2013.96

Stephen, A. M. (1995). Resistant starch. En: Kritchevsky, D. y Bonfield, C. (Eds). Dietary Fiber in Health and Disease. Minnesota: American Association of Cereal Chemists, Inc., pp.453-458.

Sun, Q., Spiegelman, D., Van Dam, R. M., Holmes, M. D., Malik, V. S., Willett, W. C. y Hu, F. B. (2010). White rice, brown rice, and risk of type 2 diabetes in US men and women. Archives of Internal Medicine 170(11), pp. 961-969.

Thomas, D. J. y Atwell, W. A. (1999). Starches. Minnesota: American Association of Cereal Chemists, Inc.

U. S. Department of Agriculture y U. S. Department of Health and Human Services (2010). Dietary Guidelines for Americans, 2010. Recuperado de https://health.gov/ dietaryguidelines/dga2010/dietaryguidelines2010.pdf

U. S. Department of Agriculture y U. S. Department of Health and Human Services (2015) Dietary Guidelines for Americans, 2015-2020. Recuperado de https://health.gov/ dietaryguidelines/2015/resources/2015-2020_Dietary_Guidelines.pdf

U. S. Department of Agriculture y U. S. Department of Health and Human Services (2016). Dietary Guidelines for Americans, 2015-2018. Recuperado de https://health.gov/ dietaryguidelines/2015/guidelines/appendix-7/

U. S. Food and Drug Administration (2016). Changes to the Nutrition Facts Label. Recuperado de https://www.fda.gov/Food/GuidanceRegulation/GuidanceDocu mentsRegulatoryInformation/LabelingNutrition/ucm385663.htm

U. S. Food and Drug Administration. (2017). Dietary Fiber. Recuperado de https://www. accessdata.fda.gov/scripts/InteractiveNutritionFactsLabel/dietary-fiber.html 
U. S. Department of Health \& Human Services y U. S. Department of Agriculture (2005). Dietary Guidelines for Americans. Washington: Government Printing Office.

Vilcanqui-Pérez, F. y Vílchez-Perales, C. (2017). Fibra dietaria: nuevas definiciones, propiedades funcionales y beneficios para la salud. Revisión. Recuperado de https://www.alanrevista.org/ediciones/2017/2/art-10/.

Zielinski, G., DeVries J. W., Craig, S. A. y Bridges, A. R. (2013). Dietary Fiber Methods in Codex Alimentarius: Current Status and Ongoing Discussions. Cereal Foods World 58(3), pp. 148-152. 